\title{
Fetal growth and the ethnic origins of type 2 diabetes
}

\author{
Michael R. Skilton
}

Received: 8 December 2014 / Accepted: 10 December 2014 / Published online: 8 January 2015

(C) Springer-Verlag Berlin Heidelberg 2015

\begin{abstract}
Birthweight is known to differ by ethnicity, with South Asian, black African and Caribbean, and Hispanic ethnic groups having lower birthweight on average, when compared with people of white European ethnicity. Birthweight is the most frequently used proxy of fetal growth, and represents the net effect of a host of genetic, physiological and pathophysiological factors. These same ethnic groups that have lower average birthweight also tend to have a higher prevalence of type 2 diabetes in adulthood. It is not unreasonable to propose that the well-established inverse association between birthweight and risk of type 2 diabetes may at least partially contribute to these differences in prevalence of type 2 diabetes between ethnic groups. This hypothesis would rely on the mechanisms that drive the ethnic differences in birthweight aligning with those that modify the risk of type 2 diabetes. In this issue of Diabetologia (DOI: 10.1007/ s00125-014-3474-7), Nightingale et al have furthered this field by determining whether ethnic differences in markers of cardio-metabolic risk are consistent with the differences in birthweight in an ethnically diverse cohort of children. The likely contribution of fetal growth to ethnic differences in risk of type 2 diabetes and cardiovascular disease is discussed, particularly in light of the magnitude of the birthweight differences, as are implications for the prevention of type 2 diabetes.
\end{abstract}

Keywords Biomarkers · Birthweight $\cdot$ Cardiovascular disease $\cdot$ Ethnic groups $\cdot$ Prevention $\cdot$ Type 2 diabetes

M. R. Skilton $(\bowtie)$

Boden Institute of Obesity, Nutrition, Exercise and Eating Disorders, The University of Sydney, D17-Charles Perkins Centre,

Sydney, NSW 2006, Australia

e-mail: michael.skilton@sydney.edu.au

\author{
Abbreviation \\ CHASE Child Heart and Health Study in England
}

The burden of type 2 diabetes is borne disproportionately by people of Asian (particularly South Asian), black African and Caribbean, and Hispanic ethnicity [1]. Socioeconomic factors, such as differences in education, employment and access to healthcare, are likely contributors, as are lifestyle factors, particularly related to dietary intake, and a genetic predisposition to increased adiposity (overall and regional) and insulin resistance [2]. Furthermore, these proposed mechanisms may interact with one another (eg. diet $\times$ gene interactions). The web is complex, and has thus far resisted efforts to ascertain with certainty the exact mechanisms responsible, and to translate this information into markedly improved outcomes.

One factor that may contribute to the ethnic differences in diabetes prevalence, yet has only attracted limited attention in this context, is birthweight. Compared with people of white European ethnicity, people of Asian, black African and Caribbean, and Hispanic ethnicity tend to have lower birthweights, and a higher prevalence of low birthweight $(<2,500 \mathrm{~g})$ [3]. There is now strong epidemiological evidence for an association between impaired fetal growth and risk of type 2 diabetes [4]. Together with hypertension, which is also more prevalent in people who were affected by impaired fetal growth, these chronic diseases both disproportionately affect certain ethnic groups [1]. Could the well-described differences in birthweight between ethnic groups at least partially account for the reciprocal differences in risk of chronic diseases in adulthood? Does the ethnic predisposition to poor cardiometabolic health have fetal origins? For hypertension, the answer appears to be a tentative yes, with differences in blood pressure between white and black adolescents being at least 
partially accounted for by differences in birthweight [5]. In the current issue of Diabetologia, Nightingale and colleagues ask the same general question, but with respect to cardiometabolic markers in childhood, using data from 3,700 children aged 9-10 years who participated in the Child Heart and Health Study in England (CHASE) [6]. CHASE participants were purposefully recruited from schools with a high proportion of students of South Asian or black African-Caribbean origin, resulting in a study population with almost equal numbers of people identifying as white European, South Asian and black African-Caribbean (note that a fourth group - 'other' - had a similar number of participants to the other three groups).

There are four key findings that progressively piece together the puzzle of whether or not differences in birthweight are likely to contribute to ethnic differences in cardio-metabolic risk markers. First, and in line with previous work, birthweight was lowest in the South Asian participants, with the white European participants having the highest birthweight. Second, key markers of cardio-metabolic health, including insulin, HOMA-IR, $\mathrm{HbA}_{1 \mathrm{c}}$, C-reactive protein, triacylglycerol, HDLcholesterol, LDL-cholesterol and diastolic blood pressure were all worse in South Asian participants compared with the white European participants. There were fewer cardiometabolic markers that differed between black AfricanCaribbean participants and white European participants. Key markers however, including insulin, HOMA-IR and $\mathrm{HbA}_{1 \mathrm{c}}$, were all worse in the black African-Caribbean participants, although to a lesser extent than those of the South Asian participants, putatively consistent with the smaller difference in birthweight. Third, there was evidence that birthweight was inversely associated with a number of cardio-metabolic risk markers, including insulin, HOMA-IR, $\mathrm{HbA}_{1 \mathrm{c}}$, urate, Creactive protein and triacylglycerol. These associations did not differ by ethnicity. The findings from these initial three steps all support the hypothesis that ethnic differences in cardio-metabolic risk have fetal origins (reflected by birthweight); however, the hypothesis falls at the fourth step. If birthweight was an important mediator of ethnic differences in cardio-metabolic health, one would expect the association to weaken with statistical adjustment for birthweight; howev$\mathrm{er}$, it steadfastly remained unchanged, with none of the ethnic differences in cardio-metabolic risk markers being materially affected. As noted by the authors, this is nonetheless consistent with the magnitude of the association of birthweight with type 2 diabetes risk, which is markedly smaller than the actual difference in diabetes rates between ethnic groups (Fig. 1). Accordingly, while there is a well-described and robust association of birthweight with risk of type 2 diabetes, which may be of relevance across the range of birthweights exhibited in any given population, it is unlikely to make a major contribution to the differences in risk of diabetes between ethnic groups, as the differences in birthweight between these groups is small compared with the population distribution.

One key consideration is that birthweight is but a marker, representing the net effect of a host of genetic, physiological and pathophysiological factors. It follows from this that the underlying mechanisms that drive the association of birthweight with cardio-metabolic biomarkers may differ from those that drive the differences in birthweight between ethnic groups. Further supporting this argument is the disconnect between ethnic differences in birthweight and rates of hypertension and heart disease in adulthood (Fig. 1). Appropriately powered longitudinal cohorts, recruited at birth, during
Fig. 1 Ethnicity, actual risk of diabetes and heart disease, and estimated risk based on birthweight. Birthweight data are approximate grams higher or lower than people of white European ethnicity; risk expressed as approximate percentage higher or lower than people of white European ethnicity $[1,4,11]$. For hypertension, actual rates are approximately $15 \%$ lower in South Asian, $45 \%$ higher in African-American and 5\% lower in Hispanic people than in people of white European ethnicity
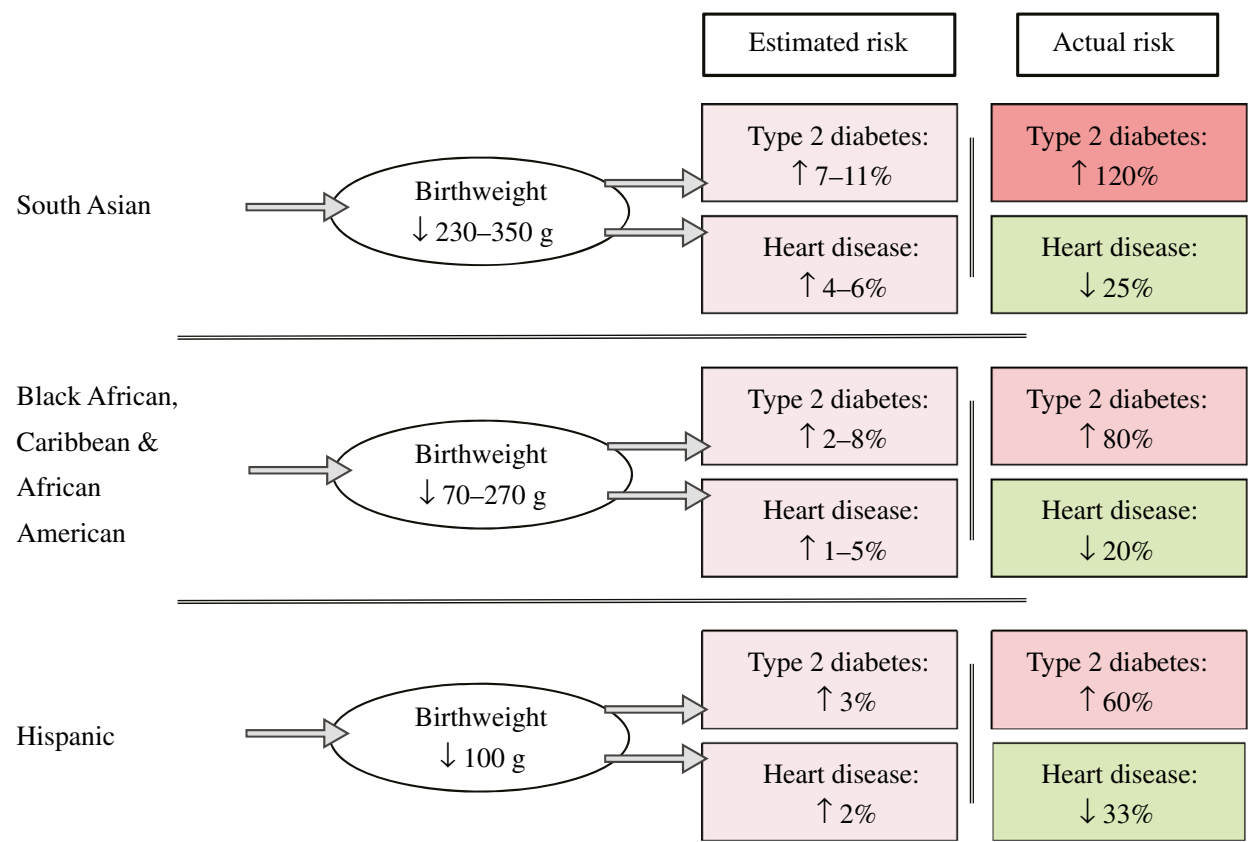
pregnancy or in the pre-conception period, are likely to inform further on these mechanisms, particularly those that include sufficient numbers of participants of diverse ethnic origins.

Nonetheless, a different outcome may be evident in adults, given additional time for the pathophysiological cardiometabolic outputs of adverse programming to develop, or if the capacity to respond to cardio-metabolic insults is reduced as a result of such programming. It would be interesting to know whether these same patterns of association are evident in the parents of the CHASE children.

The use of well-described biomarkers in childhood, as opposed to incidence or prevalence of clinical disease in adulthood, can be considered both a strength and limitation of the study. There is a reasonable biological rationale and consistency with adult measures for the use of these biomarkers in children. Their use is common for studies detailing associations with early life risk factors, partly due to the tradeoff between age of recruitment and quality of data on putative early life exposures, combined with the relatively recent interest in this area, which has come to prominence predominantly since the late 1980s. Accordingly, participants in studies with detailed early life phenotyping have generally not yet reached sufficient age for development of the main chronic diseases of adulthood. While justified on this basis, for many of these risk markers, long-term tracking is poor or not known [7]. Evidence from some of the more developed longitudinal studies originally recruited during childhood, however, supports independent associations of some biomarkers measured in childhood with type 2 diabetes risk and atherosclerotic vascular disease in adulthood [8]. The work of Nightingale and colleagues was collected during childhood, yet as part of a cross-sectional study, without the benefits of serial measures of biomarkers throughout childhood, such as those used to demonstrate that the higher systolic blood pressure in black adolescents is at least partially due to differences in birthweight [5], and without prospective detailed phenotyping of the fetus and newborn (e.g. 14\% of the birthweight data were derived from parental recall).

There remain key questions concerning the descriptive and mechanistic nature of the fetal origins of type 2 diabetes risk, that can and should be addressed through observational studies. Without evidence of effective intervention or prevention strategies, however, this field of research risks irrelevancy, particularly with regard to effective translation into clinical practice and policy. The fetal origins of chronic disease should be preventable; but the most likely strategies in the near future are unlikely to be those that target birthweight for a variety of reasons, not least of which is the fact that existing interventions are relatively ineffective at altering birthweight [9], a situation that is unlikely to change in the near future given the considerable risks to the fetus from interventions during this period. Rather, long-term 'life course' strategies aimed at preventing the chronic disease outcomes are more likely to be feasible and efficacious [10]. A similar general model should be applicable to address the ethnic differences in type 2 diabetes risk, with the development of diabetes prevention programmes tailored to specific ethnic groups. In combination with ongoing strategies to improve access to healthcare and other socioeconomic drivers of health disparities, such strategies may make significant inroads into reducing the ethnic and fetal origins of metabolic disease.

Funding MRS is supported by a National Health and Medical Research Council (NHMRC) fellowship (\#1004474).

Duality of interest The author contributed to the development of educational resources for AstraZeneca, relating to clinical management of type 2 diabetes.

Contribution statement The author was the sole contributor to this paper.

\section{References}

1. Barnes PM, Adams PF, Powell-Griner E (2008) Health characteristics of the Asian adult population: United States, 2004-2006. Adv Data: $1-22$

2. Shai I, Jiang R, Manson JE et al (2006) Ethnicity, obesity, and risk of type 2 diabetes in women: a 20 -year follow-up study. Diabetes Care 29:1585-1590

3. Shiono PH, Klebanoff MA, Graubard BI, Berendes HW, Rhoads GG (1986) Birth weight among women of different ethnic groups. JAMA 255:48-52

4. Whincup PH, Kaye SJ, Owen CG et al (2008) Birth weight and risk of type 2 diabetes: a systematic review. JAMA 300:2886-2897

5. Cruickshank JK, Mzayek F, Liu L et al (2005) Origins of the "black/ white" difference in blood pressure: roles of birth weight, postnatal growth, early blood pressure, and adolescent body size: the Bogalusa heart study. Circulation 111:1932-1937

6. Nightingale CM, Rudnicka AR, Owen CG, et al. Birthweight and risk markers for type 2 diabetes and cardiovascular disease in childhood: the Child Heart and Health Study in England (CHASE). Diabetologia. doi:10.1007/s00125-014-3474-7

7. Chen X, Wang Y (2008) Tracking of blood pressure from childhood to adulthood: a systematic review and meta-regression analysis. Circulation 117:3171-3180

8. Magnussen CG, Koskinen J, Juonala M et al (2012) A diagnosis of the metabolic syndrome in youth that resolves by adult life is associated with a normalization of high carotid intima-media thickness and type 2 diabetes mellitus risk: the Bogalusa heart and cardiovascular risk in young Finns studies. J Am Coll Cardiol 60:1631-1639

9. de Onis M, Villar J, Gulmezoglu M (1998) Nutritional interventions to prevent intrauterine growth retardation: evidence from randomized controlled trials. Eur J Clin Nutr 52(1):S83-S93

10. Skilton MR, Mikkilä V, Würtz P et al (2013) Fetal growth, omega-3 fatty acids and progression of subclinical atherosclerosis: preventing fetal origins of disease? The Cardiovascular Risk in Young Finns Study. Am J Clin Nutr 97:58-65

11. Huxley R, Owen CG, Whincup PH et al (2007) Is birth weight a risk factor for ischemic heart disease in later life? Am J Clin Nutr 85: $1244-1250$ 